\title{
Dynamics of charged gibbsite platelets in the isotropic phase
}

\author{
Dzina Kleshchanok, ${ }^{a}$ Marco Heinen, ${ }^{b}$ Gerhard Nägele, ${ }^{b}$ and Peter Holmqvist ${ }^{* b}$
}

\author{
Received Xth XXXXXXXXXX 20XX, Accepted Xth XXXXXXXXX 20XX \\ First published on the web Xth XXXXXXXXXX $200 X$ \\ DOI: $10.1039 / b 000000 x$
}

\begin{abstract}
We report on depolarized and non-depolarized dynamic light scattering, static light scattering, and steady shear viscosity measurements on interacting charge-stabilized gibbsite platelets suspended in dimethyl sulfoxide (DMSO). The average collective and (long-time) translational self-diffusion coefficients, and the rotational diffusion coefficient, have been measured as functions of the platelet volume fraction $\phi$, up to the isotropic-liquid crystal (I/LC) transition. The non-depolarized intensity autocorrelation function, measured at low scattering wavenumbers, consists of a fast and a slowly decaying mode which we interpret as the orientationally averaged collective and translational self-diffusion coefficients, respectively. Both the rotational and the long-time self-diffusion coefficients decrease very strongly, by more than two orders of magnitude, in going from the very dilute limit to the I/LC transition concentration. A similarly strong decrease, with increasing $\phi$, is observed for the inverse zero-strain limiting steady shear viscosity. With increasing $\phi$, increasingly strong shear-thinning is observed, accompanied by a shrinking of the low shear-rate Newtonian plateau. The measured diffusion coefficients are interpreted theoretically in terms of a simple model of effective charged spheres interacting by a screened Coulomb potential, with hydrodynamic interactions included. The disk-like particle shape, and the measured particle radius and thickness polydispersities, enter into the model calculations via the scattering amplitudes. The interaction-induced enhancement of the collective diffusion coefficient by more than a factor of 20 at larger $\phi$ is well captured in the effective sphere model, whereas the strong declines both of the experimental translational and rotational self-diffusion coefficients are underestimated.
\end{abstract}

\section{Introduction}

Colloidal platelets are abundant in nature (e.g., as clay minerals or red blood cells) and can be readily synthesized in the laboratory in form of mixed metal hydroxides, gibbsite, gold platelets, et cetera. The most prominent examples of colloidal platelets are various types of natural clays ${ }^{1}$, which figure in sediment transport in rivers, and in the oceans and lakes, and which are responsible for dangerous landslides ${ }^{2}$. Clays are widely used as rheological modifiers for surface coatings, paints, and drilling fluids ${ }^{3,4}$.

The rheological applications of clays are based on their microstructural properties, originating from a highly anisotropic shape and a correspondingly anisotropic particle interactions. As a necessary step towards understanding the more complex behavior of concentrated clay platelet suspensions regarding rheology, sedimentation, and sediment transport, the fundamental dynamic properties in dilute isotropic solutions must be addressed.

So far, most studies on the dynamics of clay and colloidal platelet suspensions have been focused on the non-equilibrium

${ }^{a}$ Van 't Hoff Laboratory for Physical and Colloid Chemistry, Debye Research Institute, Utrecht University, H.R. Kruytgebouw, N-701 Padualaan 8, 3584 CH Utrecht, The Netherlands

${ }^{b}$ Institute of Complex Systems (ICS-3), Forschungszentrum Jülich, D-52425 Jülich, Germany

*p.holmqvist@fz-juelich.de ergodic to non-ergodic transition in dense systems ${ }^{5-7}$, and on the properties of the non-ergodic state ${ }^{8,9}$. Only few theoretical and experimental investigations have been done so far on less concentrated, fluid-state isotropic suspensions of clays or colloidal platelets. For instance, both the translational and rotational diffusion coefficients of a single platelet were studied by simulation, and the resulting data for an extended range of aspect ratios were fitted to general polynomial expressions for cylinders by Ortega and García de la Torre ${ }^{10}$. This study allows to compare the single-platelet diffusion properties of non-interacting platelets with the results of experimental diffusion measurements, but it includes no hint on how diffusion is affected by particle interactions.

The concentration dependence of sedimentation and diffusion coefficients of uncharged, sterically interacting platelets and clays has been explored in ${ }^{11,12}$ by dynamic light scattering and ultracentrifugation. For platelets, the earlier investigations in ${ }^{11}$ were made in a rather dilute concentration regime where no effect of particle interactions on diffusion was detected. Moreover, explicit theoretical expressions have been derived for the time-dependent intensity autocorrelation function of non-interacting cylindrical particles such as platelets ${ }^{11,13-16}$ which, in principle, can be used to determine the single-platelet rotational diffusion coefficient from standard dynamic light scattering or X-ray photon correlation spectroscopy (XPCS) measurements in non-interacting parti-

This journal is @ The Royal Society of Chemistry [year]

Journal Name, 2010, [vol], 1-11 |1 
cle systems.

For rather dilute systems of uncharged polymer-grafted clay suspensions ${ }^{12}$, normalized intensity autocorrelation functions (IACFs) have been measured in vertical-vertical (VV) and vertical-horizontal $(\mathrm{VH})$ scattering geometry ${ }^{17}$. A strong slowing of the diffusive modes was found both in $\mathrm{VH}$ and in VV geometry. In VV geometry, only a single diffusive mode was detected, even though it was argued that a second, cooperative mode should be present due to the osmotic pressure of polymers grafted on the clay particles ${ }^{12}$.

In the present study, we explore how the dynamics of charge-stabilized colloidal gibbsite platelets suspended in DMSO and present in their isotropic phase, is affected by direct and hydrodynamic particle interactions. Using static light scattering (SLS) and depolarized and non-depolarized dynamic light scattering (DDLS and DLS), we study the effect of the platelet concentration on the measured translational and rotational self-diffusion coefficients, and on the collective diffusion coefficient. Moreover, the concentration- and shearrate dependence of the steady shear dispersion viscosity is determined experimentally. Our scattering and rheological experiments cover the full isotropic phase concentration regime up to the I/LC transition.

The theoretical description of the platelet dynamics in nondilute systems is severely complicated by the anisotropy in the direct ${ }^{18}$ and, to an even larger extent, by the indirect hydrodynamic interactions (HIs). No readily applicable theoretical schemes or simulation results are available for our gibbsite system. Therefore, in a first attempt to analyze theoretically the experimental trends in the diffusion data, we use a simplifying model where the direct interactions in a pair of gibbsite platelets are described approximately by the spherically symmetric, repulsive part of the Derjaguin-Landau-VerweyOverbeek (DLVO) potential ${ }^{19}$. Clays, such as Laponite, are mixed silica and metal oxides. This can lead to charges of opposite sign on their faces and edges ${ }^{20-22}$. The present gibbsite platelets consist solely of aluminium hydroxide, so that their faces and rims of gibbsite have surface charges of the same $\operatorname{sign}^{23}$. In our effective sphere model of gibbsite in DMSO, we account for the influence of the HIs but leave out the effects of interaction polydispersity. However, the disk-like shape, and the experimentally determined size polydispersity of the gibbsite platelets, are accounted for in the scattering amplitudes. The simplifying model of cylindrical platelets with spherically symmetric direct and hydrodynamic interactions permits us to take advantage of a wealth of theoretical and computer simulation results on rotational ${ }^{24,25}$ and translational self-diffusion, and collective diffusion of charged colloidal spheres ${ }^{25-27}$. The model can be expected to apply approximately to semi-dilute suspensions of isotropically dispersed platelets well below the overlap volume fraction. For larger concentrations, it is bound to fail.

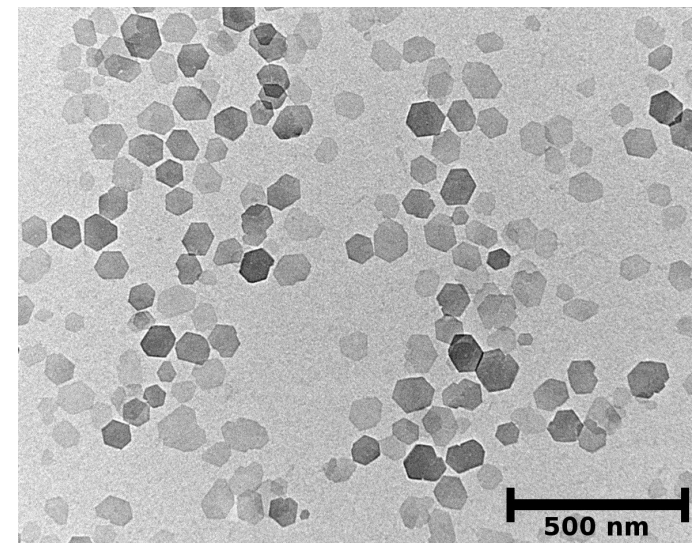

Figure 1 Transmission electron micrograph of a dried sample of gibbsite platelets used in the present study.

\section{Experimental details}

We study suspensions of hexagonal colloidal gibbsite $[\gamma-$ $\mathrm{Al}(\mathrm{OH})_{3}$ ] platelets of average radius $\langle R\rangle=44.2 \mathrm{~nm}$ and average thickness $\langle h\rangle=7.66 \mathrm{~nm}$, dispersed in dimethyl sulfoxide (DMSO). The gibbsite particles can be described approximately as a polydisperse system of thin circular cylinders of radius $R$ and (small) height $h$. We have determined the radius and height distributions from transmission electron microscopy (TEM) pictures, and using atomic force microscopy, giving polydispersities (i.e., relative standard deviations) in $R$ and $h$ of $s_{R}=17.3 \%$ and $s_{h}=55.3 \%$, respectively. An exemplary TEM picture of gibbsite platelets used in this study is shown in Figure 1.

The mean aspect ratio, $p=\langle h\rangle /(2\langle R\rangle)=0.087$, of platelets is so small that the ultrathin disk limit $(h \rightarrow 0)$ can be applied as a reasonable approximation. In this limit, one obtains $D_{t}^{0}=\left(D_{t}^{0, \|}+2 D_{t}^{0, \perp}\right) / 3=k_{B} T /\left(12 \eta_{0}\langle R\rangle\right) \approx 3.8 \times 10^{-12} \mathrm{~m}^{2} / \mathrm{s}$ for the orientationally averaged single-disk translational diffusion coefficient ${ }^{28,29}$, and $D_{r}^{0, \perp}=3 k_{B} T /\left(32 \eta_{0}\langle R\rangle^{3}\right) \approx 2.2 \times$ $10^{3} / \mathrm{s}$ for the (end-over-end tumbling) rotational diffusion coefficient determined in small- $q$ DDLS experiments on noninteracting platelets. Here, $k_{B}$ is the Boltzmann constant, $T$ is the absolute temperature, and $\eta_{0}$ is the solvent shear-viscosity. For $p \rightarrow 0, D_{r}^{0, \perp}$ becomes equal to the rotational diffusion coefficient, $D_{r}^{0, \|}$, which characterizes rotation with respect to the platelet rotational symmetry axis. A small but finite value of $p$ lowers somewhat the values of $D_{t}^{0}$ and $D_{r}^{0, \perp}$, and $D_{r}^{0, \|}$ and $D_{r}^{0, \perp}$ become different from each other. Tirado and García de la Torre ${ }^{30,31}$ provide precise polynomial fits to their simulation data for the diffusion coefficients of cylindrical platelets as a function of $p$. However, the aspect ratio range, $p>0.1$, covered by most of these fits, does not include the small aspect ratio of the present gibbsite platelets. We can alterna- 
tively estimate the effect of a non-zero $p$ using analytic results $^{32}$ for the single-particle diffusion coefficients of oblate spheroids of same $p$ and same volume $V_{p}=\pi\left\langle R^{2} h\right\rangle$, giving $D_{r}^{0, \perp}=1.4 \times 10^{3} / \mathrm{s}$ and $D_{t}^{0}=3.1 \times 10^{-12} \mathrm{~m}^{2} / \mathrm{s}$.

DMSO is a polar, aprotic, and low-viscous solvent of dielectric constant $\varepsilon=47.2$, at $T=293 \mathrm{~K}$, and viscosity $\eta_{0}=$ $2 \times 10^{-3} \mathrm{~Pa} \cdot \mathrm{s}$, in which the platelets are charge-stabilized, forming a suspension that remains transparent up to the isotropic-liquid crystal (I/LC) transition.

Different from aqueous gibbsite suspensions, which are turbid already at low concentrations, gibbsite in DMSO systems show no multiple scattering in the investigated concentration range. Indeed, the considered systems are fully transparent and the VH IACF shows no mode in addition to the rotational diffusion mode (c.f., Eq. (8)). Moreover, different from aqueous suspensions, there is no residual $\mathrm{CO}_{2}$ contamination, and no solvent self-dissociation, so that low-ionic strength systems can be easily prepared. Thus, we can use DDLS to study the rotational and translational diffusion as a function of the gibbsite number concentration up to the I/LC transition point. Our standard (D)DLS/SLS apparatus is equipped with a krypton ion laser of wavelength $\lambda_{0}=647 \mathrm{~nm}$ as a light source, and a $\lambda / 2$ plate used as polarizer and analyzer (Bernhard Halle Nachfl., Berlin, Germany). Each sample was measured both in vertical-unpolarized (VU) and VH scattering geometry, for values of the wave number, $q$, smaller than the value, $q_{m}$, where the primary peak of the mean scattered intensity, $I(q)$, occurs.

The low-shear-rate limiting steady shear viscosity (referred to as the steady-shear viscosity further down) was measured using an AR-G2 rheometer from TA instruments with a coneplate geometry $\left(\right.$ diameter $=20 \mathrm{~mm}$; cone $\left.=2^{\circ}\right)$. All measurements were performed under vapor lock.

DLS and steady-shear viscosity data were recorded for a large number of gibbsite volume fractions $\phi=M /\left(\rho_{m} V\right)$ up to the $\mathrm{I} / \mathrm{LC}$ transition at $\phi_{\mathrm{I} / \mathrm{LC}} \approx 8 \%$. Here, $M$ is the total mass of added gibbsite of known mass density $\rho_{m}$, and $V$ is the suspension volume. In addition, the steady shear viscosity, $\eta$, was measured as a function of shear rate $\dot{\gamma}$, for a large number of concentrations.

\section{Results}

\subsection{Dynamic light scattering results}

The dynamic light scattering functions in VH and VU geometry have been recorded for a large number of scattering wavenumbers and concentrations up to the volume fraction at the I/LC transition. Results for the normalized intensity autocorrelation functions (IACFs), $g_{2}^{\mathrm{VU}}(q, t)$, (filled symbols) and $g_{2}^{\mathrm{VH}}(q, t)$ (open symbols), for two different volume fractions $\phi=0.8 \%$ and $4.8 \%$, are shown in Fig. 2 . We point out here that $g_{2}^{\mathrm{VU}} \approx g_{2}^{\mathrm{VV}}$ to excellent accuracy, due to the very small optical anisotropy of the gibbsite platelets. The rather dilute system at $\phi=0.8 \%$ has only weak inter-platelet correlations, whereas the system at $\phi=4.8 \%$ is not very far from the I/LC transition.

Consider first the low-concentrated system in panel (a) of Fig. 2, where $g_{2}^{\mathrm{VU}}(q, t)-1$ and $g_{2}^{\mathrm{VH}}(q, t)-1$ are shown for four different $q$-values located well below $q_{m}$, with $q\langle R\rangle=1.26$ for the largest considered $q$. At this low concentration, both IACFs are only slightly stretched, decaying basically singleexponentially. The stretching is due here to the rather small polydispersity, $s_{R}=17.3 \%$, in the disk radius $R$. Due to the small value of $p$, the more pronounced polydispersity in $h$ of value $s_{h}=55.3 \%$ has only little influence on the particle diffusion coefficients, and on the scattering amplitudes in the experimentally accessed $q$-range where $q\langle R\rangle \lesssim 1$ and thus $q\langle h\rangle \lesssim 0.1$. The time relaxation of $g_{2}^{\mathrm{VH}}(q, t)$ is faster than that of $g_{2} \mathrm{VU}(q, t)$.

To globally account for polydispersity and particle correlation effects, which both give rise to a potentially continuous superposition of correlation times, we have fitted all of the measured functions $g_{2}(q, t)-1$ in panel (a) of Fig. 2 by the Kohlrausch-Williams-Watts (KWW) stretched exponential form, $\left\{\exp \left[-\left(t / \tau_{i}\right)^{\beta_{i}}\right]\right\}^{2}$, characterized by the overall decay times $\tau_{i}$ and stretching exponents $\beta_{i} \leq 1$. An IACF decaying nearly single-exponentially has a value of $\beta$ close to one. For example, one finds $\beta_{i} \approx 0.9$ for the IACF's of the lowconcentration systems in panel (a) of Fig. 2. The KWW mean relaxation rates $\Gamma_{i}$, with $i \in\{\mathrm{VH}, \mathrm{VU}\}$, are obtained from $\Gamma_{i}=1 /\left[\tau_{i} \times \Gamma\left(1 / \beta_{i}\right) / \beta_{i}\right]$, where $\Gamma$ denotes the gamma function $^{33}$. In all our experiments, the area coherence factor $C$ in the Siegert relation $C g_{1}^{2}(q, t)=g_{2}(q, t)-1$ is practically equal to one.

In panel (b) of Fig. 2, the resulting relaxation rates $\Gamma_{\mathrm{VH}}$ and $\Gamma_{\mathrm{VU}}$ at $\phi=0.8 \%$ are plotted as functions of $q^{2}$. At the small $q$-values considered where $q\langle R\rangle \lesssim 1$, the relaxation rates in $\mathrm{VH}$ and $\mathrm{VU} \approx \mathrm{VV}$ geometry can be expressed as $\Gamma_{\mathrm{VH}}=6 D_{r}^{\perp}+\mathcal{O}\left(q^{2}\right)$ and $\Gamma_{\mathrm{VV}}=D_{t} q^{2}+\mathcal{O}\left(q^{4}\right)$, respectively ${ }^{13,15-17,34,35}$, where $D_{t}$ and $D_{r}^{\perp}$ are interpreted as the translational and (end-over-end tumbling) rotational diffusion coefficients, respectively. In this interpretation, polydispersity effects are accounted for in an overall way through the KWW relaxation rates. Contributions to $\Gamma_{\mathrm{VV}}$ of $\mathcal{O}\left(q^{4}\right)$ which arise from rotational diffusion and rotational-translational coupling, are of no relevance for the considered small $q$-values.

Regarding the low-concentration system results in panels (a) and (b), no distinction is required between short- and longtime diffusion coefficients. The slopes and intercepts of $\Gamma_{\mathrm{VU}}$ and $\Gamma_{\mathrm{VH}}$ in panel (b), obtained from a $q^{2} \rightarrow 0$ extrapolation, are approximately equal to the zero-concentration diffusion coefficients. A zero- $\phi$ extrapolation of all investigated systems, with the relaxation rates obtained as described above, 

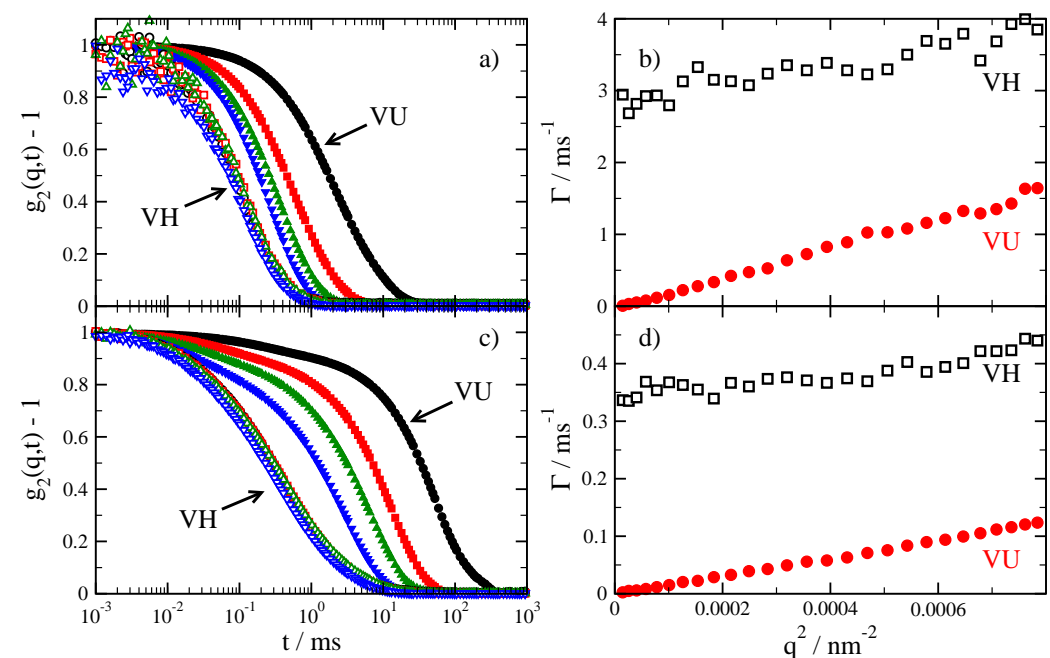

Figure 2 Panels a and c: Reduced intensity autocorrelation function, $g_{2}(q, t)-1$, versus correlation time $t$, for gibbsite in DMSO. Panels b and $\mathrm{d}$ : (mean) relaxation rates, $\Gamma_{V H}$ and $\Gamma_{V U}$, as functions of $q^{2}$. The gibbsite volume fraction is $\phi=0.8 \%$ in panels a and $\mathrm{b}$, and $\phi=4.8 \%$ in panels $\mathrm{c}$ and $\mathrm{d}$. The wavenumbers in a and c are: $q=7.6 \times 10^{-3} \mathrm{~nm}^{-1}$ (black circles), $q=1.47 \times 10^{-2} \mathrm{~nm}^{-1}$ (red squares), $q=2.08 \times 10^{-2}$ $\mathrm{nm}^{-1}$ (green triangles), and $q=2.84 \times 10^{-2} \mathrm{~nm}^{-1}$ (blue triangles). Open symbols: VH scattering geometry results. Filled symbols: $V U \approx V V$ scattering geometry results.

leads to $D_{t}^{0}=2.1 \times 10^{-12} \mathrm{~m}^{2} / \mathrm{s}$ and $D_{r}^{0, \perp}=0.34 \times 10^{3} / \mathrm{s}$. Both values are somewhat smaller than those obtained from the ultrathin platelet approximation using $R=\langle R\rangle$.

The data for $\Gamma_{\mathrm{VH}}$ in panel (b) show an overall linear increase in $q^{2}$, but are more noisy than those for $\Gamma_{\mathrm{VU}}$. This originates from the much lower scattering intensity in VH geometry, which for the gibbsite system is by a factor of $10^{-4}$ smaller than that in VU geometry. Therefore, for the present system, VU and VV measurements give practically indentical results.

At larger platelet concentrations, where direct and hydrodynamic interactions come into play, one notices interesting changes in the scattering functions. The normalized VU and VH IACFs, and the corresponding KWW relaxation rates at $\phi=4.8 \%$, are depicted in panels (c) and (d) of Fig. 2, respectively. It is apparent from panel (c) that, in VU geometry, an additional quickly relaxing mode occurs. Moreover, the decay of the slow-mode in $g_{2}^{\mathrm{VU}}(q, t)-1$ is slightly more stretched. In VH geometry, no additional mode is seen at this larger concentration, but the decay is more stretched than in the $\phi=0.8 \%$ case. These features are indicators of pronounced platelet correlations at $\phi=4.8 \%$, requiring now the distinction between short- and long-time diffusion properties. The subdiffusive transition regime is characterized by the structural relaxation time defined by $\tau_{I}^{0}=1 /\left(6 D_{r}^{0, \perp}\right) \approx 0.05 \mathrm{~ms}$.

Using again the Siegert relation, we can fit the two-mode decay of $g_{2}^{\mathrm{VU}}$ observed in panel (c) in the probed time window by the two stretched-exponential form,

$g_{2}^{\mathrm{VU}}(q, t)-1=\left[A e^{-\left(t / \tau_{t}^{\mathrm{VU}}\right)^{\beta_{t}}}+(1-A) e^{-\left(t / \tau_{c}^{\mathrm{VU}}\right)^{\beta_{c}}}\right]^{2}$,

involving the fit parameters $A, \tau_{t}^{\mathrm{VU}}, \tau_{c}^{\mathrm{VU}}, \beta_{t}$, and $\beta_{c}$ with $0 \leq A \leq 1$ and $\Gamma_{c}^{\mathrm{VU}}>\Gamma_{t}^{\mathrm{VU}}>0$. Here, $\Gamma_{i}^{\mathrm{VU}}=$ $1 /\left[\tau_{i}^{\mathrm{VU}} \times \Gamma\left(1 / \beta_{i}\right) / \beta_{i}\right]$, for $i=t, c$, and the stretching exponent $\beta_{c}$ is larger than 0.9 in all considered cases. The exponent $\beta_{t}$ is larger than 0.9 for $\phi \lesssim 2 \%$, reducing to values of $\beta_{t} \approx 0.7$ at $\phi_{\mathrm{I} / \mathrm{LC}} \approx 8 \%$. Note that, in a similar fashion, the stretching exponent $\beta_{r}$ of the rotational diffusion mode in the VH IACF fulfills $\beta_{r}>0.9$ for $\phi \lesssim 2 \%$, reducing to $\beta_{r} \approx 0.55$ at $\phi=\phi_{\mathrm{I} / \mathrm{LC}}$.

Several of the correlation functions were also fitted with a CONTIN $^{36}$ routine and first cumulant with results deviating from the above described method by no more that $5 \%$. Both the fast-mode and the slow-mode relaxation rates, $\Gamma_{c}^{\mathrm{VU}}=q^{2} D_{c}$ and $\Gamma_{t}^{\mathrm{VU}}=q^{2} D_{t}$, show the expected diffusive $q^{2}$-dependence, allowing for the determination of the associated diffusion coefficients $D_{c}$ and $D_{t}$, respectively. For $\phi=4.8 \%$, the ordering relations $D_{c}>D_{t}^{0}$ and $D_{t}<D_{t}^{0}$ are obeyed. In panel (d), $\Gamma_{t}^{\mathrm{VU}} \approx \Gamma^{\mathrm{VV}}=q^{2} D_{t}+\mathcal{O}\left(q^{4}\right)$ is plotted as a function of $q^{2}$, with $D_{t}$ inferred from the small- $q$ slope. The corresponding fast mode rate, $\Gamma_{c}^{\mathrm{VU}}$, is not shown in the figure. Its associated diffusion coefficient, $D_{c}$, has been determined as the slope of $\Gamma_{c}^{\mathrm{VU}}\left(q^{2}\right)$, extrapolated to $q=0$.

Panel (d) shows additionally the relaxation rate, $\Gamma^{\mathrm{VH}}=$ $6 D_{r}^{\perp}+D_{t} q^{2}$, of the somewhat stretched out single-exponential decay of $g_{2}^{\mathrm{VH}}(q, t)$, determined again using the KWW analysis. Note here that the small- $q$ slope of $\Gamma^{\mathrm{VH}}\left(q^{2}\right)$ is equal, within 


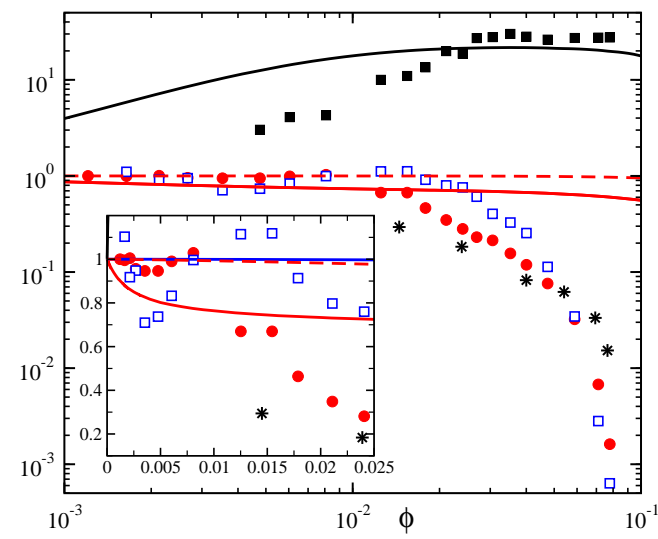

Figure 3 Volume fraction dependence of various dynamic properties including the normalized fast-mode diffusion coefficient, $D_{c} / D_{t}^{0}$, (filled squares), and the slow-mode diffusion coefficient, $D_{t} / D_{t}^{0}$, (filled circles), both obtained in VU geometry, and the normalized (mean) rotational diffusion coefficient, $D_{r}^{\perp} / D_{r}^{0, \perp}$ (open squares), obtained from VH measurements. Stars: Inverse low-shear-rate viscosity $\left(\eta / \eta_{0}\right)^{-1}$, obtained from rheometric measurements. Solid black and dashed red lines: Predictions for $D_{c} / D_{t}^{0}$ and $D_{t}^{S} / D_{t}^{0}$ within the effective charged sphere model, calculated using the corrected $\delta \gamma$ scheme. Solid blue line (inset only): Scaling relation $D_{r}^{S} / D_{r}^{0}=$ $1-1.3 \phi_{\text {eff }}^{2}$ for the short-time rotational diffusion coefficient of effective charged spheres, using $\phi_{\mathrm{eff}}=1.93 \phi$. Solid red line: $D_{t}^{L} / D_{t}^{0}$ of effective charged spheres, calculated using the simplified MCT scheme with far-field HIs correction. The inset magnifies the details at low $\phi$ on a double linear scale.

the experimental noise, to that of $\Gamma_{t}^{\mathrm{VU}}\left(q^{2}\right)$. The slope in both geometries is identified as the translational self-diffusion coefficient, $D_{t}$, of interacting platelets in the isotropic phase. Likewise, the coefficient $D_{r}^{\perp}$ determined from the zero- $q$ intercept of $\Gamma^{\mathrm{VH}}\left(q^{2}\right)$ in panel (d), obeying $D_{r}^{\perp}<D_{r}^{0, \perp}=D_{r}^{\perp}(\phi \rightarrow 0)$, can be interpreted as the rotational self-diffusion coefficient at non-zero concentrations.

The same evaluation procedure as explicated in Fig. 2 for two specific concentrations, was applied to an extended set of concentrations up to the I/LC transition value. The obtained reduced diffusion coefficients, $D_{t} / D_{t}^{0}, D_{r}^{\perp} / D_{r}^{0, \perp}$ and $D_{c} / D_{t}^{0}$, are depicted in Fig. 3 in their dependence on $\phi$. According to Fig. $3, D_{t}$ and $D_{r}^{\perp}$ remain constant, within the experimental scatter, in the lower-concentration range of $\phi \lesssim 0.5 \%$. This is the expected behavior of particles at low concentration which diffuse practically uncorrelated. On the other hand, already at $\phi=1 \%$, the fast-mode coefficient, $D_{c}$, exceeds largely its zero-concentration value $D_{t}^{0}$ whereas, as viewed on the extended vertical scale of Fig. 3, $D_{t}$ and $D_{r}^{\perp}$ are just about to start their strong decline below their respective infinite dilution values.

A concentration dependence similar to that of $D_{c}$ has been reported for the collective diffusion coefficient of polymers and flexible rod systems ${ }^{37,38}$. However, the gibbsite platelets studied in the present work include no flexible parts. Moreover, the onset of the strong increase of $D_{c}(\phi)$ is observed at concentrations far below the platelet overlap concentration, $\phi^{*}=(3 / 2) p$, of about $13 \%$. Incidentally, an alternative definition of the overlap concentration invoking the random close packing volume fraction, $f=0.64$, of monodisperse spheres given by $\rho^{*}\left(4 \pi\langle R\rangle^{3} / 3\right)=f$ results in $\phi^{*}=(3\langle h\rangle / 4\langle R\rangle) f \approx$ $8.3 \%$. This value is somewhat fortuitously close to the concentration, $\phi_{\mathrm{I} / \mathrm{LC}}=8 \%$, where the $\mathrm{I} / \mathrm{LC}$ transition is observed experimentally.

Since the dynamics of freely rotating charged platelets at concentrations well below the overlap concentration $\phi^{*}$ is most strongly influenced by the monopolar terms in the farfield electrostatic and hydrodynamic interactions, and since collective diffusion is to a large extent triggered by the osmotic compressibility, $D_{c}(\phi)$ can be expected to behave similar to the collective diffusion coefficient measured in low-salinity suspensions of spherical charged colloids ${ }^{26,27}$.

The coefficient $D_{c}$ for charge-stabilized colloidal spheres also shows a distinct rise at lower $\phi$, passing trough a maximum at an intermediate $\phi_{m}$ before it eventually decreases at higher concentrations. For homogeneous suspensions of monodisperse spheres, $D_{c}$ is the ratio of the reduced sedimentation velocity divided by the isothermal osmotic compressibility. This allows to interpret the shape of $D_{c}(\phi)$, with a maximum at $\phi_{m}$, in an intuitive physical picture: Due to the long-ranged electrostatic repulsion, the compressibility of low-salinity, charge-stabilized suspensions strongly decreases as a function of $\phi$ at low concentrations, causing a steep increase in $D_{c}(\phi)$. At higher concentration, hydrodynamic slowing of sedimentation eventually overcompensates the decrease in compressibility, leading to a maximum in $D_{c}(\phi)$ at $\phi_{m}$. With increasing salt content, the value of $D_{c}\left(\phi_{m}\right)$ decreases, whereas $\phi_{m}$ increases. The collective diffusion coefficient quantifies the relaxation of density fluctuations on macroscopic length scales. Thus, different from $D_{t}$ and $D_{r}^{\perp}$, it is relatively insensitive to the details of the direct and hydrodynamic interactions on shorter length scales. This makes it more clear why our effective sphere model prediction for $D_{c}(\phi)$ in Figure 3 agrees well with the experimental data for concentrations even up to $\phi \approx \phi_{\mathrm{I} / \mathrm{LC}}$.

We argue that Fig. 3 depicts the long-time values of the translational self-diffusion coefficient $D_{t}$ which, for interacting systems, can be substantially smaller than its short-time counterpart, whose deviations from the infinite dilution limit $D_{t}^{0}$ are comparatively small. That $D_{t}$ should be interpreted as a long-time property indeed follows from Eq. (1) and the considered small- $q$ range in combination with the observation that $D_{c}>D_{t}^{0}>D_{t}$ at $\phi \gtrsim 0.5 \%$. Due to the fast decay of the collective mode, $\Gamma_{t}^{\mathrm{VU}}$ is determined essentially by the coefficient $D_{t}$ 
for times $t \gtrsim 1 /\left(q^{2} D_{c}\right)$, which is part of the long-time regime $t \gg \tau_{I}^{0} \approx 0.05 \mathrm{~ms}$ once the zero- $q$ extrapolation is made in determining $D_{t}$. Different from self-diffusion, the long-time value of $D_{c}$ is practically equal to the short-time value, for all concentrations in the isotropic liquid state regime ${ }^{39,40}$.

\subsection{Simplifying diffusion model of platelets}

To analyze the trends in the concentration dependence of the gibbsite platelet diffusion coefficients, and to gain further support for the interpretation of $D_{c}$ as a collective diffusion coefficient, we introduce here a simplifying model. In this model, the platelets are described regarding their interactions as uniformly charged effective hard spheres of radius $a$, interacting by the repulsive electrostatic part,

$$
\beta u_{\mathrm{el}}(r>2 a)=L_{B} Z^{2}\left(\frac{e^{\kappa a}}{1+\kappa a}\right)^{2} \frac{e^{-\kappa r}}{r}
$$

of the DLVO potential ${ }^{19}$. Here $L_{B}=1.18 \mathrm{~nm}$ is the Bjerrum length of DMSO at $T=293 \mathrm{~K}, Z$ is the effective particle charge in units of the proton elementary charge, and $\kappa^{2}=4 \pi L_{B}\left[n_{\text {eff }}|Z|+2 n_{s}\right] /\left(1-\phi_{\text {eff }}\right)$ is the square of the Debye screening parameter. The latter is determined by the number concentration, $n_{s}$, of residual 1-1 electrolyte ions which we assume to be equal to $7 \mu \mathrm{M}$, and the number concentration, $n_{\text {eff }}|Z|$, of surface-released monovalent counterions. Furthermore, $n_{\text {eff }}=3 \phi_{\text {eff }} /\left(4 \pi a^{3}\right)$ is the number concentration of effective spheres. There are several alternatives to define the effective sphere radius $a$, depending on the quantity considered. In the present model, $a$ is obtained from equating the $2^{\text {nd }}$ virial coefficient of neutral spheres of radius $a$ to that of cylindrical platelets of radius $\langle R\rangle$ and height $\langle h\rangle$, assuming all orientations to be equally probable ${ }^{41}$. This results in $a=0.735\langle R\rangle=32.5 \mathrm{~nm}$. A slightly smaller value of $a=(3 \pi / 32)^{1 / 3}\langle R\rangle=29.4 \mathrm{~nm}$ would be obtained from the $2^{\text {nd }}$ virial coefficient in the zero- $h$ limit.

In addition to the DLS measurements described before, we have measured the static mean scattered intensity, $I(q)$, using SLS. Fig. 4 includes the experimentally determined intensities (open symbols), for platelet volume fractions $\phi=0.16 \%$ (black) and $0.88 \%$ (red).

Light scattering measurements are restricted to a smaller $q$ range than small angle X-ray scattering (SAXS) so that the interaction peak in $I(q)$ is resolved experimentally for the lower concentrated system only. Using the effective sphere interaction model, with $u_{\mathrm{el}}(r)$ according to Eq. (2), we have calculated $I(q)$ approximately within the rotational-translational decoupling approximation ${ }^{39,42}$, where it is given by

$$
I(q)=I_{0} \phi P_{m}(q) S_{m}(q),
$$

with the so-called measurable static structure factor,

$$
S_{m}(q)=[1-X(q)]+X(q) S(q) .
$$

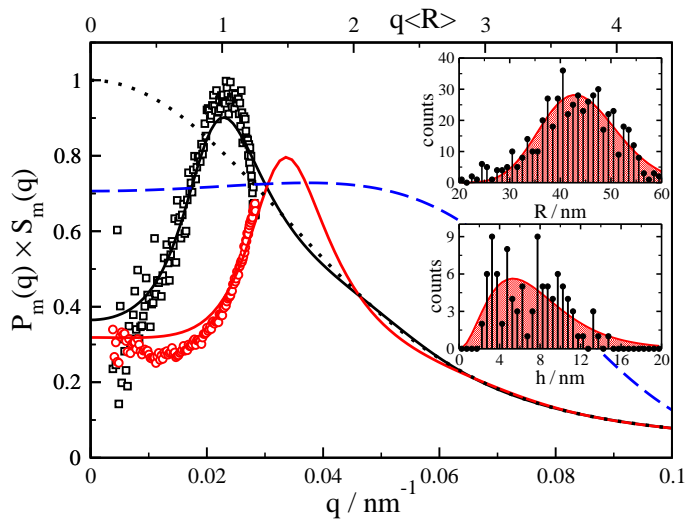

Figure 4 Normalized mean scattered intensity, $I(q) /\left(I_{0} \phi\right)$, of gibbsite platelets in DMSO, for $\phi=0.16 \%$ (black squares) and $\phi=$ $0.88 \%$ (red circles), in comparison to the theoretical fits using $\phi_{\text {eff }}=$ $0.43 \%$ (black line) and $\phi_{\text {eff }}=1.68 \%$ (red line). Black dotted curve: measurable form factor $P_{m}(q)$. Blue dashed curve: decoupling amplitude $X(q)$. Insets: experimental histograms of the platelet radius $R$ (top) and thickness $h$ (bottom). The red shaded areas are least-squares fits by unimodal Schulz distributions with mean values $\langle R\rangle=44.2 \mathrm{~nm}$ and $\langle h\rangle=7.66 \mathrm{~nm}$, and relative standard deviations of $s_{R}=17.3 \%$ and $s_{h}=55.3 \%$, respectively.

Here, $I_{0}$ is a $q$-independent factor with the dimension of intensity, which is the same in all intensity measurements corrected for source intensity, recording time, and scattering volume. Moreover, $S(q)$ is the structure factor of the monodisperse system of effective spheres interacting by the pair potential in Eq. (2). The measurable form factor, $P_{m}(q)$, and the dimensionless decoupling amplitude $X(q)$,

$$
\begin{aligned}
P_{m}(q) & =\left\langle f^{2}\right\rangle(q), \\
X(q) & =\langle f\rangle^{2}(q) / P_{m}(q),
\end{aligned}
$$

with $0 \leq X(q) \leq 1$, are given in terms of the first and second moments of the scattering amplitude, $f(q, R, h, \mu)=$ $V_{p} b(q, R, h, \mu)$, of a cylindrical platelet of volume $V_{p}=\pi R^{2} h$, where $\mu$ denotes the cosine of the angle between a platelet's rotational symmetry axis and the scattering wave vector. The simple analytic expression for the dimensionless form amplitude, $b(q, R, h, \mu)$, is given in the literature ${ }^{15,43,44}$.

Eqs. (3)-(6) are based on the assumption that the particle shape, orientation, and the size polydispersity affect the form amplitudes only, which excludes anisotropic interaction effects. Therefore, the decoupling approximation can be reasonably applied at lower concentrations only, where the platelets rotate essentially freely. The brackets, $\langle\ldots\rangle$, in Eqs. (5) and (6) represent averages with respect to the platelet orientation variable $\mu$ (assuming free rotation), and the platelet radius and height joint distribution function $p(R, h)$, which we assume to be factorisable as $p(R, h)=p_{R}(R) \times p_{h}(h)$. Histograms for the marginal distributions $p_{R}(R)$ and $p_{h}(h)$ of the dried speci- 
men, obtained from electron microscopy and atomic force microscopy, respectively, are shown in the insets of Fig. 4. On fitting unimodal Schulz distributions both to $p_{R}(R)$ and $p_{h}(h)$, we obtain mean values $\langle R\rangle=44.2 \mathrm{~nm}$ and $\langle h\rangle=7.66 \mathrm{~nm}$, at relative standard deviations of $55.3 \%$ and $17.3 \%$, respectively. Note that for $q \lesssim 2 /\langle R\rangle, X(q) \approx 0.72$ stays practically constant.

The static structure factor $S(q)$ in Eq. (4), describing pair correlations of effective charged spheres, has been calculated using the modified penetrating-background corrected rescaled mean spherical approximation (MPB-RMSA), introduced recently by part of the present authors ${ }^{45,46}$. This analytic method allows for an efficient and accurate calculation of structure factors of non-overlapping spheres, interacting by a repulsive Yukawa-type potential such as the one given in Eq. (2).

A fit of the decoupling approximation expression for $I(q)$ in Eq. (3) to the experimental intensities involves $Z, \phi_{\text {eff }}$, $I_{0}$ and, within a reasonably small range, also $n_{s}$ as the fitting parameters. Since an accurate determination of the effective particle charge number $Z$ relies on fitting the maximum in $I(q)$, not located inside the SLS-resolved $q$-range for most of the considered concentrations, we alternatively estimate $Z$ using the experimentally observed I/LC transition concentration, determined in our low-salinity experiments as $\phi_{\mathrm{I} / \mathrm{LC}}=8 \%$. Earlier simulations ${ }^{47,48}$ predict the isotropicnematic transition of neutral hard disks to occur at $n_{\mathrm{I} / \mathrm{LC}} d^{3} \approx 4$, where $n_{\mathrm{I} / \mathrm{LC}}$ is the isotropic-phase number density at the transition point, and $d$ is the disk diameter. Using this relation, we estimate the charge on a gibbsite platelet by assuming that $d=2\left[\langle R\rangle+\kappa^{-1}\right]$ and $n_{\mathrm{I} / \mathrm{LC}}=\phi_{\mathrm{I} / \mathrm{LC}} /\left(\pi\left\langle R^{2} h\right\rangle\right)$, with $\kappa^{2}=4 \pi L_{B}\left(n_{\mathrm{I} / \mathrm{LC}}|Z|+2 n_{s}\right) /\left(1-\phi_{\mathrm{I} / \mathrm{LC}}\right)$. For the considered large concentration at the I/LC transition point, the residual salt contribution to $\kappa$ can be neglected. Solving for the effective platelet charge gives the value $Z=71$. For simplicity, and since the concentration dependence of $Z$ for gibbsite in DMSO is unknown, this effective charge value is used in all our calculations, independent of $\phi$. According to ${ }^{23}, Z=71$ is a reasonable charge value for gibbsite platelets.

As demonstrated in Fig. 4, decently good fits of the experimental $I(q)$ in the probed $q$-range are obtained from adjusting the only remaining fit parameter, $\phi_{\text {eff }}$, entering into the MPBRMSA calculation of $S(q)$. The global factor $I_{0}$ in Eq. (3) only sets the overall intensity scale and is therefore system independent. Assuming a homogeneous linear relation between $\phi_{\text {eff }}$ and $\phi$, so that $\phi_{\text {eff }}=0$ for $\phi=0$, from our fit we obtain $\phi_{\text {eff }}=1.93 \phi$.

While the theoretical fit of the $\operatorname{SLS} I(q)$ is satisfying, future SAXS measurements are required to obtain $I(q)$ in a more extended $q$-range which covers all its structural features, allowing for a more detailed fitting.

The effective sphere interaction model in combination with the rotational-translational decoupling approximation is easily generalized from the SLS intensity to the normalized timedependent electric field autocorrelation function, which for small $q$ in $\mathrm{VV}(\approx \mathrm{VU})$ geometry is given by

$$
g_{1}^{\mathrm{VV}}(q, t)=[1-B] e^{-q^{2} W(t)}+B S(q \rightarrow 0) e^{-q^{2} D_{c} t},
$$

with $B=X(0) /[X(0)+(1-X(0)) S(0)]$. Here, $W(t)=$ $1 / 6<[\mathbf{r}(t)-\mathbf{r}(0)]^{2}>$ is the mean-squared displacement of a charged effective sphere at center-of-mass position $\mathbf{r}(t)$ with initial (short-time) slope $D_{t}^{S}$, and final (long-time) slope $D_{t}^{L}$, where $D_{t}^{L}<D_{t}^{S} \leq D_{t}^{0}$. Moreover, on ignoring the very small difference between long- and short-time collective diffusion coefficients, which becomes noticeable at very high concentrations only, $D_{c}$ is given by $D_{c}=D_{t}^{0} H(q \rightarrow 0) / S(q \rightarrow 0)$, where $H(q)$ is the so-called hydrodynamic function ${ }^{16,39,49}$. Eq. (7) is fully consistent with the for the gibbsite in DMSO suspensions observed two-mode decay of the IACFs, fitted using Eq. (1) with $\beta_{c} \approx \beta_{t} \approx 1$ in the concentration range where the effective sphere model applies, and with the interpretation of the experimental $D_{t}$ as a long-time self-diffusion coefficient. We note that in the simplifying model considered here, the diffusion coefficients of platelets are simply approximated by those of the effective charged spheres.

Using the effective sphere parameters $n_{s}=7 \mu \mathrm{M}, a=32.5$ $\mathrm{nm}$, and $Z=71$ determined as described above, and on varying the (effective) volume fraction in small steps using the relation $\phi_{\text {eff }}=1.93 \phi$, a set of $S(q)$ 's has been generated using the MPB-RMSA scheme, which in turn was employed as input to the otherwise parameter-free self-part corrected $\delta \gamma$ method of calculating $H(q)^{25,50-53}$. The corrected $\delta \gamma$ method includes many-body hydrodynamic interactions in an approximate way, making predictions for the $H(q)$ of Brownian spheres with Yukawa-type repulsion in overall good agreement with Stokesian Dynamics computer simulation results 52 .

Regarding the approximations invoked in our simplifying analytic model, the agreement between the calculated $D_{c}(\phi)$ and the experimental data is rather satisfying. The increase of $D_{c}$ with increasing $\phi$ to values about 20 times larger than $D_{t}^{0}$ is qualitatively captured. The theoretical $D_{c}$ reaches a shallow maximum at about $\phi=3 \%$, originating from the interplay of osmotic compressibility and sedimentation coefficient $\mathrm{H}(q \rightarrow$ $0)$ as discussed, e.g., in 26,54 .

The large- $q$ limiting value of $H(q)$ is equal to the reduced short-time self-diffusion coefficient $D_{t}^{S} / D_{t}^{0}$. The self-part corrected $\delta \gamma$-scheme result for $D_{t}^{S}(\phi)$ is included into Fig. 3 (see also the inset). In the explored $\phi$-range, $D_{t}^{S}(\phi)$ decreases only mildly with increasing $\phi$. Its $\phi$-dependence is overall well described by the form $\left(1-a_{t} \phi_{\mathrm{eff}}^{4 / 3}\right) D_{t}^{0}$ with $a_{t} \approx 2.9$, which is the typical concentration-dependence of $D_{t}^{S}$ for lowsalinity systems of charged spheres ${ }^{27}$. For $\phi \gtrsim 2 \%$, however, the decay of the experimental (long-time) $D_{t}$ in Fig. 3 with 
increasing $\phi$ is very strong. For volume fractions $\phi \gtrsim 7 \%$ near to $\phi_{\mathrm{I} / \mathrm{LC}}, D_{t}$ has decreased to values less than $1 \%$ of $D_{t}^{0}$. This is similar to the slowing down of self-diffusion seen for block copolymers in a neutral solvent when the disorderedordered transition is approached ${ }^{55}$, in polymer solutions with increasing concentration ${ }^{38}$, and also for polymer grafted clay particles $^{12}$. The measured values of $D_{t}^{L} / D_{t}^{0}$ for gibbsite are much smaller than those reached by the long-time translational diffusion coefficient of low-salinity charge-stabilized spheres in the fluid phase, which reaches its minimal value of $D_{t}^{L} / D_{t}^{0} \approx D_{t}^{L} / D_{t}^{S} \approx 0.1$ at the freezing transition volume fraction $\phi_{f} 56,57$. For the effective sphere model used here, $\phi_{f}$ can be estimated on basis of the empirical Hansen-Verlet freezing criterion, $S\left(q_{m}, \phi_{\mathrm{eff}}=\phi_{f}\right) \approx 3.1$, for charged spheres at low salinity ${ }^{58-60}$. With $S(q)$ computed in MPB-RMSA, this results in the freezing transition volume fraction $\phi_{\mathrm{eff}}=42 \%$, corresponding to $\phi=\phi_{\text {eff }} / 1.93=22 \%$. If gibbsite platelets would behave exactly like effective spheres, this would mean that they freeze into a crystalline phase at $\phi=22 \%$. The large difference in the concentrations of the I/LC transition of platelets and the fluid/crystalline transition of effective spheres, and the enormously different ratios $D_{t}^{L} / D_{t}^{S}$ at the two transitions, highlight again the failure of the effective sphere model at larger concentrations.

We attribute the strong decay of the experimental $D_{t}$ at larger $\phi$ to the uprising influence of the anisotropic electrosteric interaction parts, and to the hydrodynamic rotationaltranslational coupling effects in platelet systems. These anisotropic interaction effects, not included in the effective sphere model, cause an additional strong slowing down of the translational and rotational self-dynamics. The inset in Fig. 3 displays the experimental data for $D_{t} / D_{t}^{0}$ (and $D_{r}^{\perp} / D_{r}^{0, \perp}$ ) on a smaller concentration range $\phi \leq 0.025$, in comparison with the corrected $\delta \gamma$-scheme predictions for $D_{t}^{S} / D_{t}^{0}$, and simplified mode-coupling theory (MCT) results for $D_{t}^{L} / D_{t}^{0}$ within the effective charged sphere model. In the simplified MCT result, the enhancing influence of HIs on $D_{t}^{L}$, typical of lowsalinity systems, is accounted for ${ }^{61-63}$. Note here that the peak height, $S\left(q_{m}\right)=1.7$ of the static structure factor at $\phi_{\mathrm{I} / \mathrm{LC}}=8 \%$ is still rather close to one so that our usage of the simplified MCT solution in place of the fully self-consistent MCT solution for $D_{t}^{L}$ is justified.

The short-time rotational self-diffusion coefficient, $D_{r}^{S}$, in charged-sphere colloidal systems follows at low salinity to a good accuracy the scaling relation $D_{r}^{S} / D_{r}^{0}=1-a_{r} \phi_{\text {eff }}^{2}$, with $a_{r} \approx 1.3$, in the whole $\phi$-range covered in Fig. $3^{24,25}$. This curve is shown as the solid blue line in the inset of Fig. 3. Different from platelets with $h>0$, spheres are characterized by a single zero-concentration rotational diffusion coefficient $D_{r}^{0}=k_{B} T /\left(8 \pi \eta_{0} a^{3}\right)$. Note here that $D_{r}^{S} / D_{r}^{0}$ decreases less strongly with increasing $\phi$ than the experimental $D_{t}^{S} / D_{t}^{0}$. This difference originates from the shorter-ranged hydrodynamic self-coupling of rotational motion ${ }^{24}$. The experimental diffusion coefficients $D_{t}$ and $D_{r}^{\perp}$ depicted in Fig. 3, obey overall the same ordering relation $D_{r}^{\perp}(\phi)>D_{t}(\phi)$ as their short-time counterparts in the effective sphere model.

The low- $q$ expression for $g_{1}^{\mathrm{VH}}(q, t)$ in our simplifying model is given by (see, e.g., ${ }^{34,64}$ )

$$
g_{1}^{\mathrm{VH}}(q, t)=e^{-q^{2} W(t)} G_{r}(t)
$$

where $G_{r}(t)=<P_{2}(\hat{\mathbf{u}}(t) \cdot \hat{\mathbf{u}}(0))>$ is the rotational selfdynamic correlation function of spheres, with the optical axis of a sphere characterized by the unit vector $\hat{\mathbf{u}}$, and with $P_{2}$ denoting the $2^{\text {nd }}$-order Legendre polynomial. In the derivation of Eq. (8), it has been assumed that the translational-rotational motions of a particle are decoupled ${ }^{34}$. This decoupling is exactly valid for hydrodynamically interacting spheres at short times only. For non-spherical particles, it is an approximation even to linear order in $t$.

At short times, $G_{r}(t)=\exp \left\{-6 D_{r}^{S} t\right\}$ decays exponentially. At long times, however, $G_{r}(t)$ decays in principle nonexponentially, with an average decay rate somewhat smaller than $D_{r}^{S 34}$. While a genuine long-time rotational self-diffusion coefficient does not exist, one can define instead a mean orientational self-diffusion coefficient, $\overline{D_{r}}$, determined by the time dependence of $G_{r}(t)$ for all times. A corresponding $\overline{D_{r}}$ of platelets is shown indeed in Fig. 3, as obtained in panel (d) of Fig. 2 using the KWW analysis. The calculation of $\overline{D_{r}}$ for colloidal hard spheres in ${ }^{34}$ suggests that $\overline{D_{r}}$ is only slightly smaller than $D_{r}^{S}$, at least for smaller values of $\phi$. The mean rotational diffusion coefficient $D_{r}^{\perp}$ depicted in Fig. 3, however, decreases very strongly at larger $\phi$, to an extent comparable to that of $D_{t}$. Like for $D_{t}$, we attribute this strong decline of $D_{r}^{\perp}$ at larger $\phi$ to the strong anisotropic electro-hydrodynamic coupling of the charged platelets.

\subsection{Steady shear viscosity results}

It is interesting to note from Fig. 3 that the strong decline of the experimental $D_{t} / D_{t}^{0}$ and $D_{r} / D_{r}^{0}$ with increasing $\phi$, is qualitatively similar to the concentration dependence of the normalized inverse low shear-rate limiting viscosity, $\eta_{0} / \eta$. To obtain the low-shear rate viscosity data shown in Fig. 3, using an AR-G2 rheometer, we have measured the viscosity as a function of shear rate $\dot{\gamma}$ for various concentrations. Results of these measurements are depicted in Fig. 5, not unexpectedly showing that the gibbsite suspensions are shear-thinning, with the shear-thinning regime shifting to lower shear rates with increasing volume fraction. The low shear-rate limiting viscosity $\eta$ is identified by the (mean) Newtonian plateau value at low shear rates, with results depicted by stars in Fig. 3. For technical reasons, no reliable viscosity data have been obtained for very low shear rates $\dot{\gamma} \lesssim 1 s^{-1}$. Moreover, for 


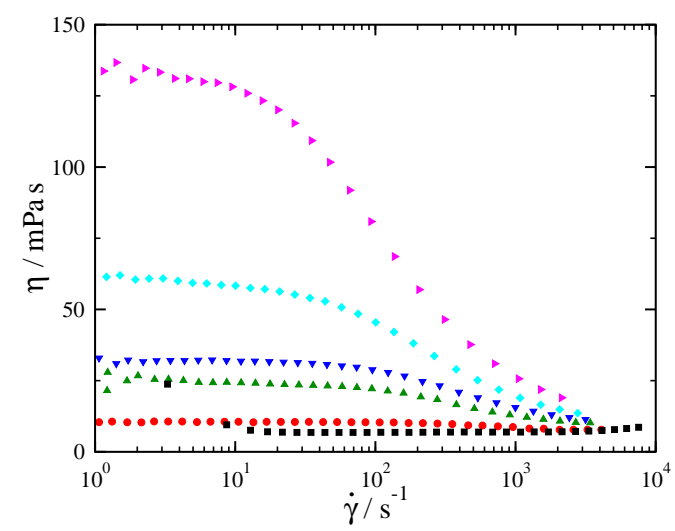

Figure 5 Shear viscosity $\eta$ of gibbsite platelets in DMSO versus shear rate $\dot{\gamma}$, for $\phi=7.6 \%, 6.9 \%, 5.4 \%, 4.0 \%, 2.4 \%$, and $1.45 \%$ (from top to bottom).

technical reasons our viscosity data do not include the lowconcentration regime from which the $\phi$-independent intrinsic viscosity, $[\eta]=\lim _{\phi \rightarrow 0}\left(\eta-\eta_{0}\right) /\left(\eta_{0} \phi\right)$, could be determined, which depends on $p$ only. For a sphere $(p=1)$ with stick hydrodynamic boundary conditions, $[\eta]=2.5$, and larger values for $[\eta]$ are obtained with increasing asphericity. From the power-law representation of the simulation data in ${ }^{10}$ for the intrinsic viscosity of thin cylinders, $[\eta] \approx 6.8$ is obtained for $p=0.087$. This value is similar to the one observed in ${ }^{65}$. Note that a spheroid of equal aspect ratio has a distinctly larger intrinsic viscosity of $[\eta] \approx 9.1$.

\section{Conclusions}

Using (D)DLS, SLS and viscometry, we have determined the VU and VH intensity autocorrelation functions, the scattered intensity $I(q)$, and the steady-shear dispersion viscosity of charged Gibbsite platelets in DMSO at low ionic strength. The experiments cover the concentration range from very dilute systems up to the I/LC transition. Our usage of DMSO as a solvent with a refractive index close to that of gibbsite, has enabled us to determine the low- $q$ dynamic scattering functions without the necessity of invoking elaborate X-ray photon correlation spectroscopy measurements.

We have provided a clear identification of the (partially stretched) fast and slow modes in the VU and VH measurements, with the associated long-time translational collectiveand self-diffusion coefficients $D_{c}$ and $D_{t}^{L}$, and the mean rotational diffusion coefficient $D_{r}^{\perp}$. A fast relaxation mode in the dynamic scattering data has been frequently reported in relation to the liquid-glass transition, the glassy state and also for polymer coated clays $6,8,66$. In the present study on rigid and non-permeable gibbsite platelets, an essentially singleexponential fast mode in the VU intensity autocorrelation function is found also in the isotropic phase. We have identified it with the collective diffusion mode characterized by $D_{c}$. Furthermore, we have provided arguments, both experimentally and theoretically, that the coefficients $D_{t}$ and $D_{r}^{\perp}$ obtained in our modes analysis, should be identified, respectively, with the translational long-time and the mean rotational self-diffusion coefficients of gibbsite platelets.

The most striking experimental observation is the extraordinarily strong decrease of the translational and rotational self-diffusion coefficients in the pre-transitional concentration regime from $\phi \approx 2 \%$ to $\phi_{\mathrm{I} / \mathrm{LC}} \approx 8 \%$. The strong decline of these coefficients with increasing concentration is accompanied by a similarly strong decline of the inverse zero-shearrate steady-shear viscosity. Examinations of possible generalized Stokes-Einstein relations between viscosity and diffusion coefficients in concentrated platelet fluids could be the topic of a future study based on the present work. As we have discussed in detail, the dramatic decrease of the experimental self-diffusion coefficients can not be understood on basis of a simplifying effective sphere model, for the reason that the translational-rotational coupling of isotropic spheres is very weak in comparison to the platelets.

Different from the translational and rotational self-diffusion coefficients, the experimental $D_{c}$ varies only little in the pretransitional concentration regime, with values about twenty times those of the single-particle translational diffusion coefficient. Similar to suspensions of charge-stabilized colloidal spheres at low ionic strength, the $D_{c}$ of gibbsite grows initially with increasing concentration before traversing a flat maximum (or plateau-like region).

The experimental findings for the diffusion coefficients have been compared with theoretical predictions by a simplifying effective sphere model where the direct platelet interactions are approximated by a spherically symmetric electrosteric repulsion of DLVO type. The effect of the hydrodynamic sphere-sphere interactions is fully accounted for in our model calculations.

The effective particle charge was estimated from the I/LC transition concentration as being $Z=71$. This charge value was kept constant in our model calculations, independent of particle concentration and salinity. An unambiguous determination of $Z$ in its dependence on $\phi$ and $n_{s}$ would require a broader $q$-range than accessible by SLS which, except for very low gibbsite concentrations, covers wavenumbers only below the principal peak position of $I(q)$. An extended wavenumber range can be probed in future SAXS and XPCS measurements. At low concentrations and within the low- $q$ regime accessed by SLS, $I(q)$ is well reproduced in our simplifying decoupling method where correlations between particle positions and orientations are neglected.

The effective sphere model gives the correct trends followed by the experimentally determined transport coefficients 
of gibbsite. In particular, the behavior of $D_{c}$ is reasonably well described over the probed concentration range, and the ordering in the magnitudes of the reduced translational and rotational self-diffusion coefficients is correctly predicted. The effective sphere model is severely more inaccurate in its quantitative predictions regarding $D_{t}^{L}$ and $D_{r}^{\perp}$, which both are more sensitive to the anisotropic direct and hydrodynamic interactions than the collective diffusion coefficient. The dramatic decay of the self-diffusion coefficients to less than $1 \%$ of their respective infinite dilution values near $\phi_{\mathrm{I} / \mathrm{LC}}$ is not reproducible in the sphere model, where values $D_{t}^{L} \gtrsim 0.1 \times D_{t}^{0}$ are predicted throughout the fluid-phase regime.

A more refined future modeling of gibbsite in DMSO should account for the shape-dependent direct interaction contributions to $I(q)$ on using, e.g., the well-established PRISM model ${ }^{67-70}$. However, regarding dynamic quantities, it will be very difficult to include the shape-dependent hydrodynamic interactions without resorting to numerically very expensive multiparticle collision, fluctuating lattice Boltzmann or Stokesian Dynamics simulations. It will be also relevant to consider in more detail the interrelated effects of size, shape and interaction polydispersity. From an experimental viewpoint, future X-ray or neutron scattering studies which cover a broader wavenumber range, and frequency-resolved viscosity measurements, will further advance our understanding of anisotropically interacting colloidal particles.

\section{Acknowledgments}

P. Davidson and H.H. Wensink are thanked for enlightening discussions. The work of D.K. was financed by the Foundation for Fundamental Research on Matter (FOM), which is part of the Netherlands Organization for Scientific Research (NWO). M.H. acknowledges support by the International Helmholtz Research School of Biophysics and Soft Matter (IHRS BioSoft). G.N. acknowledges funding from the Deutsche Forschungsgemeinschaft (SFB-TR6, project B2).

\section{References}

1 Handbook of clay science, Elsevier, Amsterdam, 2006, p. 1224.

2 A. Khaldoun, P. Moller, A. Fall, G. Wegdam, B. De Leeuw, Y. Meheust, J. O. Fossum and D. Bonn, Phys. Rev. Lett., 2009, 103, 188301.

3 G. C. Maitland, Curr. Opin. Colloid Interface Sci., 2000, 5, 301-311.

4 H. H. Murray, Appl. Clay Sci., 2000, 17, 207-221.

5 S. Mossa, C. De Michele and F. Sciortino, J. Chem. Phys., 2007, 126, 014905.

6 T. Nicolai and S. Cocard, J. Colloid Interface Sci., 2001, 244, 51-57.

7 B. Ruzicka, L. Zulian and G. Ruocco, Phys. Rev. Lett., 2004, 93, 258301.

8 B. Abou, D. Bonn and J. Meunier, Phys. Rev. E, 2001, 64, 021510.

9 R. Bandyopadhyay, D. Liang, H. Yardimci, D. A. Sessoms, M. A. Borthwick, S. G. J. Mochrie, J. L. Harden and R. L. Leheny, Phys. Rev. Lett., 2004, 93, 228302.
10 A. Ortega and J. García de la Torre, J. Chem. Phys., 2003, 119, 9914 9919.

11 F. M. van der Kooij, A. P. Philipse and J. K. G. Dhont, Langmuir, 2000, 16, 5317-5323.

12 D. Shah, G. Fytas, D. Vlassopoulos, J. Di, D. Sogah and E. P. Giannelis, Langmuir, 2005, 21, 19-25.

13 W. Eimer, J. R. Williamson, S. G. Boxer and R. Pecora, Biochemistry, 1990, 29, 799-811.

14 R. Pecora, J. Chem. Phys., 1968, 49, 1036.

15 S. Fujime and K. Kubota, Biophys. Chem., 1985, 23, 1-13.

16 J. K. G. Dhont, An Introduction to Dynamics of Colloids, Elsevier, Amsterdam, 1996.

17 B. J. Berne and R. Pecora, Dynamic Light Scattering: With Applications to Chemistry, Biology, and Physics, Wiley, New York, 1st edn., 1976.

18 D. G. Rowan, J.-P. Hansen and E. Trizac, Mol. Phys., 2000, 98, 13691378.

19 E. J. W. Verwey and J. T. G. Overbeek, Theory of the Stability of Lyophobic Colloids, Elsevier, New York, 1948.

20 L. Harnau, Mol. Phys., 2008, 106, 1975-2000.

21 L. Li, L. Harnau, S. Rosenfeldt and M. Ballauff, Phys. Rev. E, 2005, 72, 051504.

22 S. L. Tawari, D. L. Koch and C. Cohen, J. Colloid Interface Sci., 2001, 240, 54-66.

23 Y. Gan and G. V. Franks, Langmuir, 2006, 22, 6087-6092.

24 G. H. Koenderink, H. Y. Zhang, D. G. A. L. Aarts, M. P. Lettinga, A. P. Philipse and G. Nägele, Faraday Discuss., 2003, 123, 335-354.

25 A. J. Banchio and G. Nägele, J. Chem. Phys., 2008, 128, 104903.

26 J. Gapinski, A. Wilk, A. Patkowski, W. Häussler, A. J. Banchio, R. Pecora and G. Nägele, J. Chem. Phys., 2005, 123, 054708.

27 M. Heinen, P. Holmqvist, A. J. Banchio and G. Nägele, J. Appl. Crystallogr., 2010, 43, 970-980.

28 J. Happel and H. Brenner, Low Reynolds Number Hydrodynamics, Springer Netherlands, 1st edn., 1983.

29 S. Kim and S. J. Karilla, Microhydrodynamics: Principles and Selected Applications, Butterworth-Heinemann, Boston, 1st edn., 1991.

30 M. Tirado, M and J. García de la Torre, J. Chem. Phys., 1979, 71, 25812587.

31 M. Tirado, M and J. García de la Torre, J. Chem. Phys., 1980, 73, 19861993.

32 F. Perrin, J. Phys. Radium, 1936, 7, 1-11.

33 C. P. Lindsey and G. D. Patterson, J. Chem. Phys., 1980, 73, 3348-3357.

34 V. Degiorgio, R. Piazza and R. B. Jones, Phys. Rev. E, 1995, 52, $2707-$ 2717.

35 N. K. Reddy, J. Pérez-Juste, I. Pastoriza-Santos, P. R. Lang, J. K. G. Dhont, L. M. Liz-Marzán and J. Vermant, ACS Nano, 2011, 5, 49354944.

36 S. W. Provencher, Comput. Phys. Commun., 1982, 27, 229-242.

37 M. Adam and M. Delsanti, Macromolecules, 1977, 10, 1229-1237.

38 U. Zettl, S. T. Hoffmann, F. Koberling, G. Krausch, J. Enderlein, L. Harnau and M. Ballauff, Macromolecules, 2009, 42, 9537-9547.

39 G. Nägele, Phys. Rep., 1996, 272, 216-372.

40 E. Wajnryb, P. Szymczak and B. Cichocki, Physica A, 2004, 335, 339358.

41 A. Isihara, J. Chem. Phys., 1950, 18, 1446-1449.

42 M. Kotlarchyk and S. H. Chen, J. Chem. Phys., 1983, 79, 2461-2469.

43 J. S. Pedersen, Adv. Colloid Interface Sci., 1997, 70, 171-210.

44 J. M. Deutch, Macromolecules, 1981, 14, 1826-1827.

45 M. Heinen, P. Holmqvist, A. J. Banchio and G. Nägele, J. Chem. Phys., 2011, 134, 044532.

46 M. Heinen, P. Holmqvist, A. J. Banchio and G. Nägele, J. Chem. Phys., 2011, 134, 129901. 
47 D. Frenkel and R. Eppenga, Phy. Rev. Lett., 1982, 49, 1089-1092.

48 J. A. C. Veerman and D. Frenkel, Phy. Rev. A., 1992, 45, 5632-5648.

49 R. B. Jones and P. N. Pusey, Annu. Rev. Phys. Chem., 1991, 42, 137-169.

50 C. W. J. Beenakker and P. Mazur, Physica A, 1983, 120, 388-410.

51 C. W. J. Beenakker, Physica A, 1984, 128, 48-81.

52 M. Heinen, A. J. Banchio and G. Nägele, submitted. preprint available at arXiv:1109.1163v1 [cond-mat.soft], 2011.

53 U. Genz and R. Klein, Physica A, 1991, 171, 26-42.

54 M. Heinen, F. Zanini, F. Roosen-Runge, D. Fedunová, F. Zhang, M. Hennig, T. Seydel, R. Schweins, M. Antalík, G. Nägele and F. Schreiber.

55 P. Holmqvist, S. Pispas, N. Hadjichristidis, G. Fytas and R. Sigel, Macromolecules, 2003, 36, 830-838.

56 H. Löwen, T. Palberg and R. Simon, Phys. Rev. Lett., 1993, 70, 15571560.

57 A. J. Banchio, G. Nägele and J. Bergenholtz, J. Chem. Phys., 2000, 113, 3381-3396.

58 M. J. Stevens and M. O. Robbins, J. Chem. Phys., 1993, 98, 2319-2324.

59 J.-P. Hansen and L. Verlet, Phys. Rev., 1969, 184, 151.

60 K. Kremer, M. O. Robbins and G. S. Grest, Phys. Rev. Lett., 1986, 57, 2694-2697.

61 G. Nägele and P. Baur, Europhys. Lett., 1997, 38, 557-562.

62 G. Nägele and P. Baur, Physica A, 1997, 245, 297-336.

63 K. Zahn, J. M. Méndez-Alcaraz and G. Maret, Phys. Rev. Lett., 1997, 79, 175-178.

64 H. Y. Zhang and G. Nägele, J. Chem. Phys., 2002, 117, 5908-5920.

65 F. M. van der Kooij, E. S. Boek and A. P. Philipse, J. Colloid Interface Sci., 2001, 235, 344-349.

66 S. Jabbari-Farouji, E. Eiser, G. H. Wegdam and D. Bonn, J. Phys. Cond.Mat., 2004, 16, L471-L477.

67 J.-P. Hansen and I. R. McDonald, Theory of Simple Liquids, Academic Press, London, 2nd edn., 1986.

68 H. C. Andersen and D. Chandler, J. Chem. Phys., 1970, 53, 547.

69 K. S. Schweizer and J. G. Curro, Adv. Chem. Phys., 1997, 98, 1-142.

70 L. Harnau and J.-P. Hansen, J. Chem. Phys., 2002, 116, 9051-9057. 